\title{
Circulating monocytes and B-lymphocytes in neovascular age-related macular degeneration
}

\author{
This article was published in the following Dove Press journal: \\ Clinical Ophthalmology \\ 17 January 2017 \\ Number of times this article has been viewed
}

\author{
Sven Magnus Hector' \\ Torben Lykke Sørensen ${ }^{1,2}$ \\ 'Clinical Eye Research Unit, Zealand \\ University Hospital, Roskilde, \\ ${ }^{2}$ Faculty of Health and Medical \\ Sciences, University of Copenhagen, \\ Copenhagen, Denmark
}

Background: Individuals with neovascular age-related macular degeneration (AMD) have altered number and distribution of retinal macrophages and show changes in circulating antibodies. We wanted to investigate the corresponding precursors, with subpopulations. We therefore measured monocyte and B-lymphocyte populations in individuals with neovascular AMD.

Design: This was an observational case-control study.

Participants or samples: A total of 31 individuals with neovascular AMD and 30 healthy age-matched controls were included.

Methods: Patients and controls were interviewed, and ophthalmological examination included visual acuity assessment using the Early Treatment Diabetic Retinopathy Study (ETDRS) chart, spectral domain optical coherence tomography (SD-OCT), slit-lamp examination and fundus photography. Moreover, venous blood was drawn and prepared for flow cytometry. Cells were gated and measured for surface markers.

Main outcome measures: Relative amounts of monocytes and B-lymphocytes with subsets, as well as selected surface markers, were measured.

Results: The two groups did not significantly differ in age, smoking history, body mass index, physical activity or C-reactive protein (CRP). Total monocytes (percentage of all leukocytes) were lower in the neovascular AMD group (median 5.5\%) compared with the level in the control group $(6.5 \%$; $P$-value: 0.028$)$. The percentage of intermediate monocytes positive for cluster of differentiation $11 \mathrm{~b}(\mathrm{CD} 11 \mathrm{~b})$ was lower for AMD patients $(99.4 \%)$ compared with $100 \%$ for the control group ( $P$-value: 0.032$)$.

Conclusion: We observed lower numbers of monocytes, which show a potentially impaired ability to migrate across the endothelial wall in patients with neovascular AMD. These subtle changes could potentially lead to an imbalance in the recruitment of macrophages into the retina during disease development.

Keywords: macular degeneration, choroidal neovascularization, flow cytometry

\section{Introduction}

Age-related macular degeneration (AMD) is the leading cause of vision impairment globally, but the cause of the disease is still unknown. The role of the immune system in AMD pathogenesis is currently under investigation by several groups, and a previous study has reported the potentially important role of monocytes in AMD pathogenesis, even though the role of both resident macrophages and peripheral monocytes is still not known. ${ }^{1}$

The peripheral monocyte population is functionally divided into three subtypes of monocytes: classical monocytes (cluster of differentiation [CD]14++CD16-), generally considered scavenger cells; intermediate monocytes (CD14++CD16+), which are
Correspondence: Sven Magnus Hector Clinical Eye Research Unit, Zealand University Hospital, Køgevej 7-I3, 4000 Roskilde, Denmark

Tel +4546323200

Email sven.hector@gmail.com
Clinical Ophthalmology 2017:1 I 179-184

179 
involved in angiogenesis and formation of reactive oxygen species; and nonclassical monocytes (CD14+CD16++), which are considered proinflammatory cells. ${ }^{2}$ Functionally, the classical and intermediate monocytes are more phagocytic than nonclassical monocytes. Distinct roles of the different monocyte subsets in human disease have been suggested, with CD16+ monocytes playing a role in autoimmune and chronic inflammatory diseases such as diabetes and obesity. ${ }^{3}$ AMD is clearly a disease of the elderly; hence, the effect of aging on monocyte function could be important in AMD. Overall, there seems to be a tendency toward impaired ability by monocytes to phagocytize in the elderly. ${ }^{4}$ This decrease in phagocytic ability could theoretically result in increased debris accumulation in the retina, leading to parainflammation.

The role of B-lymphocytes has not previously been studied in relation to AMD, but it has been suggested that antiretinal antibodies may play a role in the etiology of AMD. ${ }^{5}$ B-lymphocytes are circulating cells that, when activated, can turn into plasma cells. Plasma cells are mostly concentrated in the lymphoid tissue and the spleen, where they produce large quantities of antibodies, which are released into the circulation. It is known that the B-lymphocyte population changes with age. ${ }^{6,7}$ This could be due to increased autoimmunity seen in the aged population. Furthermore, mammalian naïve B-lymphocytes express a complement factor $\mathrm{H}$-like protein that has similar properties as factor $\mathrm{H}$, a part of the complement cascade known to affect the development of AMD. ${ }^{8,9}$

Therefore, we wanted to study the monocyte and Blymphocyte populations in patients with neovascular AMD and compare our findings with healthy, age matched controls.

\section{Methods}

\section{Design, setting and participants}

During a 10-month period, we included 61 participants in this case-control study. All participants were more than 70 years of age and nonsmokers. Patients with AMD ( $\mathrm{n}=31)$ typically were asked to participate during their regular assessment visits. Most patients were included during ongoing anti-vascular endothelial growth factor (VEGF) treatment, but none had received ranibizumab or aflibercept within 4 or 8 weeks respectively. All patients had previously been diagnosed with neovascular AMD by a medical retinal specialist using fluorescein and indocyanine green angiography, spectral domain optical coherence tomography (SD-OCT) and clinical examination. We also asked relatives as well as patients who arrived at the department for reasons other than retina-related illness to participate as a control group $(n=30)$.

\section{Interview, examination and sampling}

Participants were asked if they had other somatic illnesses and were more specifically asked if they currently suffer or had suffered from hypertension, coronary disease, diabetes or known renal insufficiency. Thereafter, all medications were recorded and participants were asked if they had any signs of ongoing infection. We also recorded alcohol and tobacco consumption. Weight and height were recorded to calculate body mass index (BMI). Finally, participants were asked if they currently participated in any regular activity to improve or maintain their physical activity.

Visual acuity of all participants was measured using the Early Treatment Diabetic Retinopathy Study (ETDRS) chart. To assess retinal pathology, we scanned all participants with SD-OCT, and for control participants, we also performed fundus photography.

Finally, we collected two tubes of venous blood. The first tube was of $3 \mathrm{~mL}$ capacity and contained lithium-heparin gel for measuring $\mathrm{C}$-reactive protein (CRP). The second tube measured $5 \mathrm{~mL}$ and contained ethylenediaminetetraacetic acid (EDTA) for flow cytometry. Analysis was performed within 4 hours of phlebotomy.

\section{Exclusion}

We excluded control participants who had dry AMD or any participants who had a CRP level greater than $22 \mathrm{mg} / \mathrm{L}$ or symptoms of infectious disease. We also excluded individuals who took medications that significantly influenced the immune system such as methotrexate and prednisolone. Individuals suffering from chronic inflammation as well as smokers or passive smokers were also excluded.

\section{Flow cytometry}

For flow cytometry, the white blood cell (WBC) count was determined using the Sysmex XE-5000 hematology analyzer (Sysmex Corporation, Kobe, Japan). A volume corresponding to $10 \times 10^{6}$ cells was transferred to a $50 \mathrm{~mL}$ polypropylene tube. The erythrocytes in the sample were lysed using Red Blood Cell Lysis Buffer, which was afterward removed by washing and centrifuging three times. The samples were centrifuged at $500 \times g$ for 5 minutes at every washing cycle. A solution corresponding to 500,000 cells was transferred to four test tubes and was incubated together with suitable amounts of fluorochromeconjugated antibody combinations. Negative isotope control panels were used to correct for unspecific binding. Flow cytometry was performed within 4 hours of sampling. The Canto II was used to record all lymphocytic events (identified by forward/ side scatter) over a period of 3 minutes for every tube. 

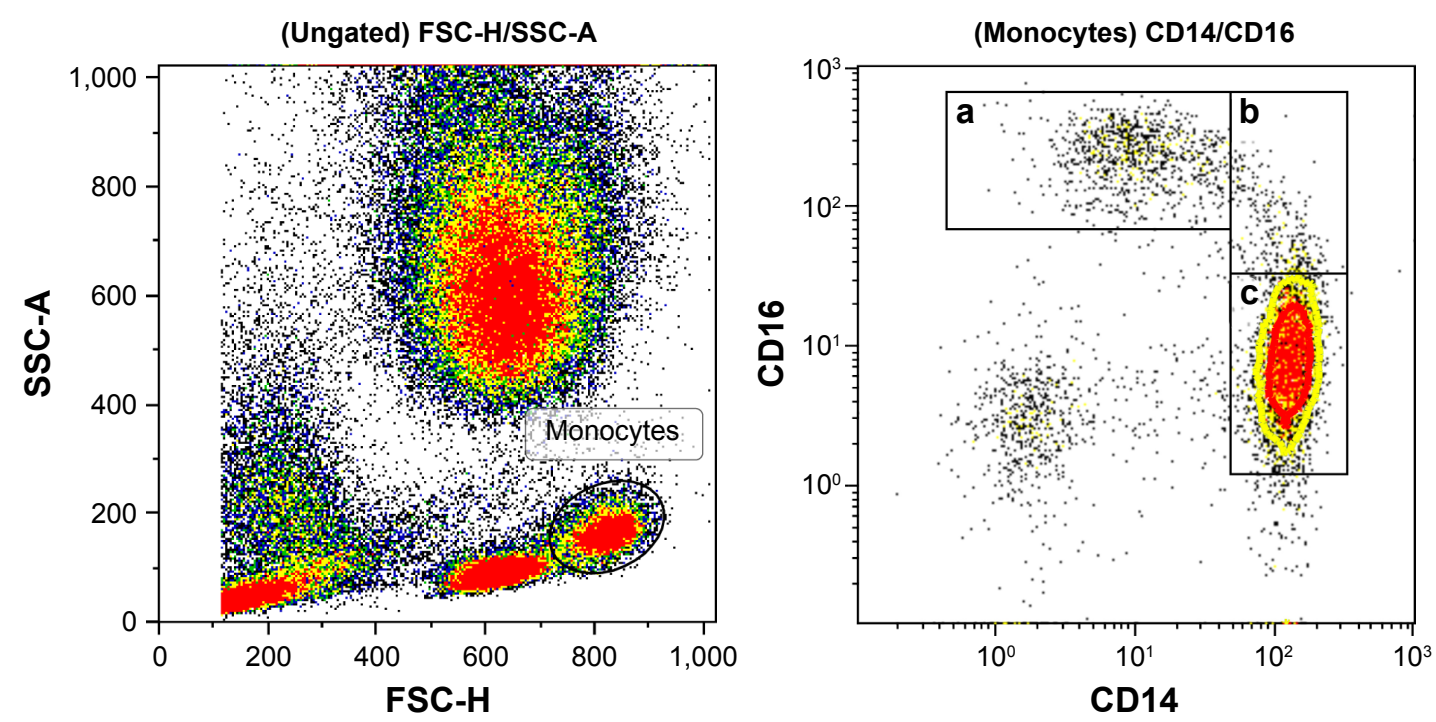

Figure I Gating strategies for monocytes and subgroups.

Notes: (a) Nonclassical monocytes; (b) intermediate monocytes; (c) classical monocytes.

Monocytes were identified by forward/side scatter and divided into subgroups using CD14 (BioLegend clone: hcd14) and CD16 (BioLegend clone: B73.1) (Figure 1). Classical monocytes were defined as CD14++ and CD16-. Nonclassical monocytes were identified as CD14+, CD16++ and intermediate ones as CD14++ and CD16+. As the three subsets seem to have different physiological functions, we wanted to study the expression of molecules for cell-cell communication on their surface, their ability to activate other leukocytes and molecules for penetrating the capillary walls and their capacity for migration to the surrounding tissue. For cell-cell communication, we chose CD11a (BioLegend Clone: HI111) and CD162 (BioLegend Clone: KPL-1). Furthermore CD63 (BioLegend Clone: H5C6), CD66B (BioLegend Clone: G10F5) and CD182 (BioLegend Clone: 5E8/CXCR2) were used as markers for the monocyte population's ability to activate neutrophils and platelets. CD62L (BioLegend Clone: DREG-56) and CD162 (BioLegend Clone: KPL-1) are both involved in leukocyte rolling on activated endothelium. CD11B (BioLegend Clone: ICRF44), CD31 (BioLegend Clone: WM59) and CD54 (BioLegend Clone: HA58) are associated with the transport of leukocytes across the endothelium and out into the inflamed tissue.

To identify B-lymphocytes, all lymphocytes in the forward/side scatter were selected and only CD19 (BioLegend Clone: HIB19)-positive cells were singled out for further characterization. To study the abundance of different subtypes of B-lymphocytes, we chose regulatory B-lymphocytes (B-regs), naïve B-lymphocytes and memory
B-lymphocytes. B-regs were defined as CD19+ (BioLegend Clone: HIB19) CD24+ (BioLegend Clone: ML5) CD38+ (BioLegend Clone: HB-7), naïve B-lymphocytes as CD19+ CD10- (BioLegend Clone: HI10a) CD27- (BioLegend Clone: M-T271) CD38- and memory B-lymphocytes as CD19+ CD10- CD27+ CD38-. A novel subgroup of B-lymphocytes called aging B-cells (ABCs) was identified as CD19+ CD11 c+ (BioLegend Clone. 3.9) CD21-(BioLegend Clone: Bu32).

\section{Statistical analyses}

Sample size power calculations using a significance level of 0.05 and a power of $80 \%$ suggested that at least 26 individuals needed to be included in each group. Statistical analyses were performed using IBM SPSS Statistics version 19. Independent-samples Mann-Whitney $U$ test was used for continuous variables and Pearson chi-squared test was used for categorical values.

\section{Ethics, consent and permissions}

After receiving written and verbal information about the purpose and execution of the study, all participants signed a consent form. They were informed of their right to withdraw at any point. The study was approved by the Regional Committee of Ethics in Research of the Region of Zealand (SJ-142).

\section{Results}

Baseline characteristics of patients and healthy individuals are shown in Table 1. Sixty-three individuals agreed to 
Table I Population characteristics

\begin{tabular}{|c|c|c|c|}
\hline Characteristics & $\begin{array}{l}\text { Patients with } \\
\text { AMD }(n=3 I)\end{array}$ & $\begin{array}{l}\text { Control } \\
\text { individuals } \\
(n=30)\end{array}$ & $P$-value \\
\hline Age, years, mean (SD) & $79.7(6.0)$ & $77.2(6.5)$ & $0.131^{\S}$ \\
\hline Males, n (\%) & $10(32)$ & $16(53)$ & $0.096^{\#}$ \\
\hline BMI, kg/m², mean (SD) & $26.6(5.1)$ & $25.9(4.6)$ & $0.555^{\S}$ \\
\hline Previous smoker, $\mathrm{n}(\%)$ & $16(52)$ & $16(53)$ & $0.893^{\#}$ \\
\hline Excessive alcohol use, $n(\%)$ & $7(23)$ & $8(27)$ & $0.71 I^{\#}$ \\
\hline Physically active, $n(\%)$ & $9(29)$ & $7(23)$ & $0.613^{\#}$ \\
\hline CRP level, mg/L, $n(\%)^{\dagger}$ & & & $0.270^{\#}$ \\
\hline$<3.1$ & $20(67)$ & $24(83)$ & \\
\hline $3.1-8.0$ & $8(27)$ & $3(10)$ & \\
\hline$>8.0$ & $2(7)$ & $2(7)$ & \\
\hline $\begin{array}{l}\text { White blood cells, } \\
\text { in } \times 10^{9} \text { cells } / \mathrm{L} \text {, mean }(\mathrm{SD})^{\ddagger}\end{array}$ & $6.4(1.6)$ & $6.1(1.3)$ & $0.430^{\S}$ \\
\hline
\end{tabular}

Notes: ${ }^{\S}$ Independent samples $t$-test; ${ }^{\#} \chi^{2}$ test; ${ }^{\dagger}$ data missing for one patient with AMD and one control individual; ${ }^{\ddagger}$ data missing for one patient with AMD and three control individuals.

Abbreviations: AMD, age-related macular degeneration; BMI, body mass index; CRP, C-reactive protein.

participate in the study. Two individuals in the control group were excluded because they were found to have dry AMD, leaving 31 in the neovascular AMD group and 30 in the control group. The neovascular group consisted of ten males and 21 females, and the control group consisted of 16 males and 14 females. This difference was not significant. The two groups did not differ significantly in age, BMI, smoking history, alcohol intake, physical activity or CRP, as well as in the WBC count.

The neovascular AMD group had significantly lower relative amounts of monocytes compared with the control group, because among all leukocytes, only $5.5 \%$ were monocytes in the neovascular AMD group, compared with $6.5 \%$ in the control group $(P=0.028)$. To further investigate whether the lower number of monocytes could be attributed to a certain subtype of monocytes, we studied the distribution of classical monocytes, intermediate monocytes and nonclassical monocytes (Figure 1). We found no differences between patients with AMD and healthy controls (Table 2), suggesting

Table 2 Total monocytes and subpopulation distribution of monocytes, expressed as percentage (interquartile range)

\begin{tabular}{llll}
\hline Population of cells & $\begin{array}{l}\text { Patients with } \\
\text { AMD }\end{array}$ & $\begin{array}{l}\text { Control } \\
\text { individuals }\end{array}$ & P-value $^{\S}$ \\
\hline Total monocytes $^{\dagger}$ & $5.5(4.3-6.5)$ & $6.5(5.3-7.3)$ & 0.028 \\
Classical monocytes $^{\ddagger}$ & $68.5(60.8-71.1)$ & $69.4(66.8-75.9)$ & 0.509 \\
Intermediate monocytes $^{\ddagger}$ & $5.1(3.1-7.2)$ & $5.0(3.0-7.1)$ & 0.088 \\
Nonclassical monocytes $^{\ddagger}$ & $9.6(5.5-12.1)$ & $8.8(6.3-11.6)$ & 0.900
\end{tabular}

Notes: ${ }^{\S}$ Mann-Whitney $U$ test; ${ }^{\dagger}$ percentage of total leukocytes; ${ }^{\ddagger}$ percentage of total monocytes.

Abbreviation: AMD, age-related macular degeneration.
Table 3 Recruitment molecules on subpopulation of monocytes, expressed as median percentage positive for marker (interquartile range)

\begin{tabular}{|c|c|c|c|c|}
\hline $\begin{array}{l}\text { Cell } \\
\text { population }\end{array}$ & Marker & $\begin{array}{l}\text { Patients with } \\
\text { AMD }\end{array}$ & $\begin{array}{l}\text { Control } \\
\text { individuals }\end{array}$ & $P$-value $\S$ \\
\hline Classical & CDIla & $100(100-100)$ & $100(100-100)$ & 1.000 \\
\hline \multirow[t]{4}{*}{ monocytes } & CDIIb & $98.6(94.6-99.9)$ & $99.4(95.1-99.9)$ & 0.432 \\
\hline & CD62L & $19.2(3.6-53.5)$ & $19.4(6.0-70.3)$ & 0.501 \\
\hline & CD54 & 99.5 (97.8-99.8) & 99.5 (98.5-99.8) & 0.896 \\
\hline & CDI62 & $100(100-100)$ & $100(100-100)$ & 1.000 \\
\hline Intermediate & CDIIa & $100(100-100)$ & $100(100-100)$ & 0.317 \\
\hline \multirow[t]{4}{*}{ monocytes } & CDIIb & $99.4(97.7-100)$ & $100(99.5-100)$ & 0.032 \\
\hline & CD62L & $15.9(8.7-34.7)$ & $13.0(5 . \mid-42.6)$ & 0.971 \\
\hline & CD54 & $100(99.2-100)$ & $99.9(99.4-100)$ & $0.87 \mid$ \\
\hline & CDI62 & $100(100-100)$ & $100(100-100)$ & 0.325 \\
\hline Nonclassical & CDIla & $100(100-100)$ & $100(100-100)$ & 0.317 \\
\hline \multirow[t]{4}{*}{ monocytes } & CDIIb & $41.9(34.9-54.3)$ & $48.0(32.9-58.5)$ & 0.363 \\
\hline & CD62L & $1.6(0.9-3.0)$ & $5.0(0.9-8.6)$ & 0.152 \\
\hline & CD54 & $98.8(98.1-99.6)$ & $98.8(97.2-99.3)$ & 0.773 \\
\hline & CDI62 & $100(100-100)$ & $100(100-100)$ & 0.309 \\
\hline
\end{tabular}

Note: $\$$ Mann-Whitney $U$ test. Bold values are statistically significant.

Abbreviations: AMD, age-related macular degeneration; CD, cluster of differentiation.

that the observed difference is due to a general decrease in the relative number of monocytes rather than a decline in a specific subgroup.

The expression of CD200 on CD11b+ monocytes has previously been reported to be increased in patients with AMD. ${ }^{1}$ Therefore, we studied a spectrum of recruitment molecules appearing on the subgroups of monocytes. We found that $\mathrm{CD} 11 \mathrm{~b}$ on intermediate monocytes was significantly reduced in the neovascular AMD group $(P<0.032)$, while no other significant differences were observed (Table 3 ).

In AMD, circulating B-cells have not been studied before, despite the previously described circulating retinal autoantibodies in patients with AMD. ${ }^{5}$ In addition, a new subpopulation of B-cells, $\mathrm{ABCs}$, has been discovered and we therefore wanted to study the distribution of B-lymphocytes and, in particular, the $\mathrm{ABC}$ population in AMD. However, no

Table 4 B-lymphocytes and subgroups, expressed as median percentage (interquartile range)

\begin{tabular}{llll}
\hline Population of cells & $\begin{array}{l}\text { Patients with } \\
\text { AMD }\end{array}$ & $\begin{array}{l}\text { Control } \\
\text { individuals }\end{array}$ & P-value $^{\S}$ \\
\hline Total B-lymphocytes $^{\dagger}$ & $6.7(4.9-9.8)$ & $6.1(4.5-9.7)$ & 0.603 \\
Memory B-lymphocytes $^{\ddagger}$ & $13.4(7.9-27.3)$ & $18.6(12.2-26.0)$ & 0.327 \\
Naïve B-lymphocytes $^{\ddagger}$ & $65.3(49.9-77.9)$ & $58.7(52.1-70.4)$ & 0.367 \\
Regulatory B-lymphocytes $^{\ddagger}$ & $50.7(41.7-68.1)$ & $45.6(36.5-58.4)$ & 0.103 \\
Aging B-lymphocytes $^{\ddagger}$ & $1.0(0.4-1.8)$ & $1.5(0.8-3.5)$ & 0.132 \\
\hline
\end{tabular}

Notes: \$Mann-Whitney $U$ test; ${ }^{\dagger}$ percentage of total lymphocytes; ${ }^{\ddagger}$ percentage of total B-lymphocytes.

Abbreviation: AMD, age-related macular degeneration. 
significant differences in the distribution of B-lymphocytes between the two groups were found (Table 4).

\section{Discussion}

In a postmortem study, Lad et al showed that patients with AMD had a significantly higher number of retinal macrophages within the macula compared with controls. ${ }^{10}$ The macrophages were also morphologically different in the two groups and some were shown to be bone marrow-derived macrophages. The mechanism behind this accumulation is not known, but it is hypothesized that oxidative stress causes impairment in the retina's ability to repair damaged tissue. ${ }^{11}$ This leads to accumulation of amyloid- $\beta$ and other waste products, as well as the formation of drusen. As a result, the retinal pigment epithelium (RPE) is damaged due to inflammation around the growing drusen. A damaged RPE, together with normal aging, leads to the thickening of Bruch's membrane and the subsequent breakdown of the bloodretina barrier, exposing the retina to the peripheral immune system. When circulating monocytes migrate over to retinal tissue, they change into macrophages, phagocytotic cells. ${ }^{1}$ Parainflammation, together with the recruitment of peripheral monocytes, orchestrates a proinflammatory environment, ultimately leading to the production of VEGF, resulting in choroidal neovascularization (CNV) and growth. ${ }^{11}$ This suggested that peripheral changes could potentially be important in AMD pathogenesis, and previous studies have shown specific profiles of monocytes in patients with AMD. ${ }^{12}$

Indeed, after experimentally induced $\mathrm{CNV}$ in mice, it has been shown that bone marrow-derived macrophages migrate to the retina from the peripheral circulation. ${ }^{13}$ But their role in relation to $\mathrm{CNV}$ is debated. The diameter and thickness of the CNV was significantly smaller in mice that did not have a normal monocyte population, the precursors to macrophages. ${ }^{14}$

On the other hand, intraocular injection of macrophages from young experimental animals, into old animals, protected the retina from the occurrence of CNV. Injection of macrophages from older animals did not have a protective effect. ${ }^{15}$ There are two main types of macrophages. M1 are proinflammatory and are tumor suppressors. M2 are stimulated by interleukin (IL)-10, are mostly anti-inflammatory and stimulate the development of new vessels and remodeling of tissue. ${ }^{16}$

As mentioned earlier, monocytes can be divided into functional subgroups. Classical monocytes are CD16 dim and produce the majority of reactive oxygen; these seem to be specialized in protection against microbial attacks. ${ }^{17}$ The intermediate subset is highly activated and is functionally not a mix of classical and nonclassical monocytes. Nonclassical and intermediate monocytes are associated with chronic inflammation in an aging population and may leave the elderly with impaired ability for phagocytosis. The nonclassical monocytes are thought to have a patrolling function. ${ }^{18,19}$

We have previously reported on the frequency of the different monocyte subtypes in AMD using the chemokine receptors CCR2 and CX3CR $1 .{ }^{20}$ No differences were observed among the different groups of patients. ${ }^{20}$ Our current results are in agreement with this finding using the CD16 molecule for monocyte subtype distinction. If our results can be confirmed in future studies, it may indicate that patients with AMD have a lower absolute number of monocytes, with no changes in the relative distribution. A lower number of monocytes that have an impaired ability to travel across the endothelium may induce neovascularization in individuals who develop wet AMD.

The intermediate subset of monocytes is more effective at antigen presentation and they express more $\mathrm{CD} 11 \mathrm{~b}$ than other monocytes, which also indicates a higher level of activation. $\mathrm{CD} 11 \mathrm{~b}$ is involved in transendothelial migration. ${ }^{18}$ Perivascular resident retinal macrophages express $\mathrm{CD} 11 \mathrm{~b}$, and if $\mathrm{CD} 11 \mathrm{~b}$ is blocked experimentally, macrophages are prevented from being recruited over activated endothelium. ${ }^{21}$

Individuals with AMD in our study showed lower levels of total monocytes and the intermediate subset expressed lower levels of the recruitment molecule CD11b. Individuals with AMD do not seem to have the ability to clear waste products efficiently from tissues, such as the retina, and may therefore be more prone to develop drusen. A recent study also found an increased number of CD11b+ cells in the peripheral blood of patients with AMD. ${ }^{22}$ Curiously, we also found that the CD200 molecule - a regulator of microglial activity - on CD11+ monocytes, was increased in patients with AMD compared to controls, suggesting the notion of an important role for CD11 in AMD pathogenesis. ${ }^{1}$

Even though retinal autoantibodies have been detected systemically in patients with AMD, the role of B-cells has not been studied in AMD. ${ }^{5}$ This is probably mostly due to the focus on the innate immune system in AMD pathogenesis but also because knowledge of the ABCs has been evolving slowly. Currently, the B-lymphocytes can be divided into regulatory B-lymphocytes (B-regs), naïve B-lymphocytes, memory B-lymphocytes and a novel subgroup called aging B-cells (ABCs). ${ }^{7}$ The role of the different B-cells in aging and disease remains to be clarified. B-cells are mostly involved in autoimmune diseases and $\mathrm{ABCs}$ seem to increase in numbers 
in aging patients with known autoimmune disease. Because AMD, as such, is not considered an autoimmune disease, it is not surprising that we do not detect any differences between patients and healthy individuals.

The number of participants in this study is relatively small, and we only gathered data from one visit for each participant. In addition, even though our findings may be statistically significant, a potential biological relevance needs to be further investigated because the observed differences are small.

\section{Conclusion}

We found that the absolute number of monocytes is diminished in patients with neovascular AMD, and in particular, we found lower levels of expression of the CD11b molecule on intermediate monocytes, suggesting a potential role for this molecule in disease development.

\section{Disclosure}

The authors report no conflicts of interest in this work.

\section{References}

1. Singh A, Falk MK, Hviid TVF, Sørensen TL. Increased expression of $\mathrm{CD} 200$ on circulating CD11b+ monocytes in patients with neovascular agerelated macular degeneration. Ophthalmology. 2013;120(5):1029-1037.

2. Xiong H, Pamer EG. Monocytes and infection: modulator, messenger and effector. Immunobiology. 2015;220(2):210-214.

3. Poitou C, Dalmas E, Renovato M, et al. CD14dimCD16+ and $\mathrm{CD} 14+\mathrm{CD} 16+$ monocytes in obesity and during weight loss relationships with fat mass and subclinical atherosclerosis. Arterioscler Thromb Vasc Biol. 2011;31(10):2322-2330.

4. Puchta A, Naidoo A, Verschoor CP, et al. TNF drives monocyte dysfunction with age and results in impaired anti-pneumococcal immunity. PLoS Pathog. 2016;12(1):e1005368.

5. Adamus G, Chew EY, Ferris FL, Klein ML. Prevalence of anti-retinal autoantibodies in different stages of Age-related macular degeneration. BMC Ophthalmol. 2014;14:154.

6. Duggal NA, Upton J, Phillips AC, Sapey E, Lord JM. An age-related numerical and functional deficit in $\mathrm{CD} 19(+) \mathrm{CD} 24(\mathrm{hi}) \mathrm{CD} 38$ (hi) B cells is associated with an increase in systemic autoimmunity. Aging Cell. 2013; 12(5):873-881.
7. Rubtsov AV, Rubtsova K, Fischer A, et al. Toll-like receptor 7 (TLR7)driven accumulation of a novel $\mathrm{CD} 11 \mathrm{c}^{+} \mathrm{B}$-cell population is important for the development of autoimmunity. Blood. 2011;118(5):1305-1315.

8. Erdei A, Julen N, Marschang P, Feifel E, Kerekes K, Dierich MP. A novel, complement factor $\mathrm{H}$-related regulatory protein expressed on the surface of human B cell lines. Eur J Immunol. 1994;24(4):867-872.

9. Grassi MA, Folk JC, Scheetz TE, Taylor CM, Sheffield VC, Stone EM. Complement factor $\mathrm{H}$ polymorphism p. Tyr402His and cuticular Drusen. Arch Ophthalmol. 2007;125(1):93-97.

10. Lad EM, Cousins SW, Van Arnam JS, Proia AD. Abundance of infiltrating $\mathrm{CD} 163+$ cells in the retina of postmortem eyes with dry and neovascular age-related macular degeneration. Graefes Arch Clin Exp Ophthalmol. 2015;253(11):1941-1945.

11. Ambati J, Atkinson JP, Gelfand BD. Immunology of age-related macular degeneration. Nat Rev Immunol. 2013;13(6):438-451.

12. Singh A. Systemic changes in neovascular age-related macular degeneration. Dan Med J. 2014;61(6):B4872.

13. Caicedo A, Espinosa-Heidmann DG, Piña Y, Hernandez EP, Cousins SW. Blood-derived macrophages infiltrate the retina and activate Muller glial cells under experimental choroidal neovascularization. Exp Eye Res. 2005;81(1):38-47.

14. Espinosa-Heidmann DG, Suner IJ, Hernandez EP, Monroy D, Csaky KG, Cousins SW. Macrophage depletion diminishes lesion size and severity in experimental choroidal neovascularization. Invest Ophthalmol Vis Sci. 2003;44(8):3586-3592.

15. Kelly J, Ali Khan A, Yin J, Ferguson TA, Apte RS. Senescence regulates macrophage activation and angiogenic fate at sites of tissue injury in mice. J Clin Invest. 2007;117(11):3421-3426.

16. Mantovani A, Sica A, Sozzani S, Allavena P, Vecchi A, Locati M. The chemokine system in diverse forms of macrophage activation and polarization. Trends Immunol. 2004;25(12):677-686.

17. Wong KL, Tai JJ, Wong WC, et al. Gene expression profiling reveals the defining features of the classical, intermediate, and nonclassical human monocyte subsets. Blood. 2011;118(5):e16-e31.

18. Hearps AC, Martin GE, Angelovich TA, et al. Aging is associated with chronic innate immune activation and dysregulation of monocyte phenotype and function. Aging Cell. 2012;11(5):867-875.

19. Seidler S, Zimmermann HW, Bartneck M, Trautwein C, Tacke F. Age-dependent alterations of monocyte subsets and monocyte-related chemokine pathways in healthy adults. BMC Immunol. 2010;11:30.

20. Falk MK, Singh A, Faber C, Nissen MH, Hviid T, Sørensen TL. CX3CL1/CX3CR1 and CCL2/CCR2 chemokine/chemokine receptor complex in patients with AMD. PLoS One. 2014;9(12):e112473.

21. Patarroyo M. Adhesion molecules mediating recruitment of monocytes to inflamed tissue. Immunobiology. 1994;191(4-5):474-477.

22. Lechner J, Chen M, Hogg RE, et al. Alterations in circulating immune cells in neovascular age-related macular degeneration. Sci Rep. 2015;5: 16754 .
Clinical Ophthalmology

\section{Publish your work in this journal}

Clinical Ophthalmology is an international, peer-reviewed journal covering all subspecialties within ophthalmology. Key topics include: Optometry; Visual science; Pharmacology and drug therapy in eye diseases; Basic Sciences; Primary and Secondary eye care; Patient Safety and Quality of Care Improvements. This journal is indexed on Submit your manuscript here: http://www.dovepress.com/clinical-ophthalmology-journal

\section{Dovepress}

PubMed Central and CAS, and is the official journal of The Society of Clinical Ophthalmology (SCO). The manuscript management system is completely online and includes a very quick and fair peer-review system, which is all easy to use. Visit http://www.dovepress.com/ testimonials.php to read real quotes from published authors. 